\title{
Derivação e validação de um modelo preditivo do perímetro abdominal
}

Cristina Marques, ${ }^{*}$ Rodrigo Neves, ${ }^{* *}$ Frederico Rosário***

\section{RESUMO}

Objectivo: analisar a relação entre o perímetro abdominal e as características dos utentes, e desenhar um modelo preditivo do perímetro abdominal.

Tipo de estudo: observacional, transversal, analítico.

Local: Unidades de Saúde Familiar (USF) Eça e Cuidados de Saúde Integrados Seixal; Unidade de Cuidados de Saúde Personalizados (UCSP) Quinta da Lomba.

População: utentes com $\geq 20$ anos, utilizadores da consulta destas unidades de saúde.

Métodos: Amostra de conveniência, de utentes que frequentaram a consulta das USF entre Maio e Julho de 2009, e da UCSP entre Fevereiro e Abril de 2010. Os utentes foram caracterizados quanto a: idade, altura, peso, género e perímetro abdominal. A amostra colhida nas USF foi aleatoriamente dividida em dois grupos: dois terços para elaboração do modelo, um terço para determinação da validade interna. A elaboração do modelo foi efectuada com base num modelo de regressão linear múltipla, método stepwise, com probabilidade de entrada de $5 \%$ e saída de $10 \%$. A amostra colhida na UCSP foi utilizada para validação externa.

Resultados: a amostra das USF foi constituída por 493 utentes, 55,6\% da USF Eça, 64,3\% do género feminino. Média de idades 57,7 $\pm 0,8$ anos, altura média $162 \pm 0,4 \mathrm{~cm}$, IMC médio $27,3 \pm 0,2 \mathrm{~kg} / \mathrm{m}^{2}$, e perímetro abdominal médio 93,8 $\pm 0,5 \mathrm{~cm} . \mathrm{A}$ comparação dos grupos de modelação e validação interna não revelou diferenças significativas pelo que se consideraram homogéneos. Elaborou-se um modelo preditivo do perímetro abdominal em que todas as variáveis se revelaram significativas, com um $\mathrm{R}^{2}=0,772$. O modelo apresentou validade interna de $92,7 \%$. A aplicação do modelo ao grupo de validação externa, constituído por 240 utentes, mostrou que $93,8 \%$ destes tinham o seu perímetro abdominal no intervalo de $95 \%$ de confiança predito pelo modelo.

Conclusões: As características individuais dos utentes podem ser utilizadas para estimar os seus perímetros abdominais.

Palavras-Chave: Perímetro Abdominal; Risco; Doenças Cardiovasculares; Modelo Linear.

\section{INTRODUÇÃO}

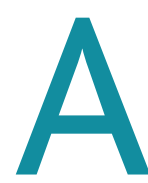
s doenças cardiovasculares constituem a principal causa de morte em Portugal, tendo em 2009 sido responsáveis, de acordo com os dados do Instituto Nacional de Estatística, por $31,9 \%$ das mortes. ${ }^{1}$ Nos últimos anos o excesso de peso/obesidade tem ganho especial importância como factor de risco cardiovascular, estimando-se que este factor de risco possa ser responsável por até $7 \%$ dos gastos em saúde. ${ }^{2}$

* Interna de Medicina Geral e Familiar, USF CSI, Seixal.

**Interno de Medicina Geral e Familiar, USF Eça, Barreiro.

***Assistente de Medicina Geral e Familiar, UCSP Quinta da Lomba, Barreiro.
Tradicionalmente usado como marcador de risco cardiovascular, o índice de massa corporal (IMC) tem sido criticado por reflectir tanto o tecido adiposo como a massa magra. ${ }^{3}$ A evidência actual sugere que as complicações vasculares encontradas no utente obeso estão mais relacionadas com a localização do tecido adiposo do que com o seu total corporal, tendo-se encontrado uma forte relação entre a adiposidade intra-abdominal e o risco cardiovascular. ${ }^{4}$

A visão tradicional do tecido adiposo visceral como simples depósito de energia tem sido substituída pela de um verdadeiro órgão endócrino com efeitos autócrinos, parácrinos e sistémicos. ${ }^{5}$ Foram já identificadas 
diversas substâncias segregadas pelo tecido adiposo visceral que podem explicar a frequente associação deste com outros factores de risco, como por exemplo o angiotensinogénio, as angiotensinas I e II, e a hipertensão arterial, ou a resistina e a resistência à insulina, entre outras. ${ }^{5} \mathrm{O}$ reconhecimento da associação do excesso de obesidade abdominal com o risco cardiovascular e metabólico ${ }^{6,7}$ levou à sua inclusão na determinação do risco cardiovascular global nas normas de orientação clínica da Sociedade Europeia de Cardiologia (ESC), que sugere para a sua avaliação a medição do perímetro abdominal (PA). ${ }^{8} \mathrm{~A}$ inclusão desta variável na avaliação do risco cardiovascular parece ganhar ainda maior importância se for tida em conta a altura do indivíduo. ${ }^{6,7,9,10}$ A medição antropométrica da obesidade abdominal é simples, barata e acessível, estando fortemente correlacionada com as medidas radiológicas usadas para a sua determinação, como por exemplo a tomografia computorizada, a ressonância magnética ou a absorsiometria radiológica de dupla energia. . $, 6,8,10$

Para alcançar o PA desejado, o utente deve ser aconselhado a adoptar estilos de vida saudáveis, especialmente no que diz respeito a uma dieta equilibrada e à prática regular de exercício físico., ${ }^{8,11}$ As metas preconizadas para o PA são diferentes consoante o género do utente, no entanto não têm em conta outras características, como por exemplo a idade ou estatura, que comprovadamente influenciam o PA..$^{4,12}$ Assim, este estudo teve como objectivos determinar a influência que o IMC, a idade, a altura, a massa corporal e o género têm sobre o PA através da derivação e validação de um modelo preditor do PA com aplicabilidade na prática clínica que permita uma intervenção personalizada visando a sua redução.

\section{MÉTODOS}

Estudo observacional, transversal e analítico, realizado na Unidade de Cuidados de Saúde Personalizados (UCSP) Quinta da Lomba e Unidade Saúde Familiar (USF) Eça, pertencentes ao Agrupamento de Centros de Saúde (ACES) Arco Ribeirinho, e na USF Cuidados de Saúde Integrados (CSI), pertencente ao ACES Seixal-Sesimbra.

A recolha dos dados decorreu em dois períodos: o primeiro, realizado na USF Eça e na USF CSI entre Maio e Julho de 2009, visou a elaboração do modelo prediti- vo e sua validação interna; o segundo período, realizado na UCSP Quinta da Lomba entre Fevereiro e Abril de 2010, teve como intuito a validação externa do modelo.

O estudo teve como população alvo os utentes com 20 ou mais anos de idade das instituições acima referidas. Foi colhida uma amostra de conveniência referente aos utentes que frequentaram a consulta de Saúde do Adulto nos períodos de recolha dos dados.

Os utentes foram caracterizados quanto a: idade (anos), género (categorizado em masculino e feminino), altura (centímetros), massa corporal (kg) e PA (centímetro, medido com fita métrica numa linha paralela ao solo, passando pelos pontos médios situados entre o bordo inferior da última costela e o bordo superior da crista ilíaca antero-superior) ${ }^{8}$ Com base nos dados recolhidos foi calculada a variável IMC $\left(\mathrm{kg} / \mathrm{m}^{2}\right)$ através da equação:

$\operatorname{IMC}\left(\mathrm{kg} / \mathrm{m}^{2}\right)=$ Massa corporal $(\mathrm{kg}) /$ altura $^{2}(\mathrm{~m})$

A avaliação das variáveis antropométricas foi efectuada por avaliação única com os instrumentos de medida disponíveis nos consultórios de cada médico. A recolha dos dados foi realizada durante o tempo de consulta pelos médicos que aceitaram participar no estudo.

Os dados, depois de recolhidos e codificados, foram transferidos para uma matriz inserida na aplicação informática Microsoft Excel $^{\circledR} 2003 .{ }^{13} \mathrm{O}$ tratamento dos dados foi efectuado com recurso aos softwares $\mathrm{R}^{\oplus}$ 2.9.2. ${ }^{14}$ e SPSS ${ }^{\circledR} 15.0$ para Windows (SPSS Inc., Chicago IL). ${ }^{15}$

A primeira amostra, colhida na USF Eça e USF CSI, foi dividida em dois grupos de forma aleatória através da geração de uma sequência de números aleatórios: dois terços foram utilizados para a elaboração do modelo preditivo; um terço para a sua validação interna. A homogeneidade destas duas amostras foi testada através dos testes $\chi^{2}$ de Pearson para as variáveis categóricas e $t$ de Student para duas amostras independentes para as variáveis contínuas. Para a tomada de decisão adoptou-se o nível de significância de $5 \%$.

A importância das variáveis estudadas foi determinada através do modelo de regressão linear múltipla, método stepwise, com probabilidade de entrada de $5 \%$ e saída de $10 \%$. 
As validades interna e externa foram avaliadas pela percentagem de utentes das amostras de validação cujo perímetro abdominal medido se encontrava dentro do intervalo de confiança de $95 \%$ predito pelo modelo. As validades foram comparadas através do teste $\chi^{2}$ para comparação de proporções.

\section{RESULTADOS}

\section{Análise Descritiva}

A primeira amostra, compreendendo os grupos para elaboração do modelo preditivo e validação interna, contabilizou um total de 493 utentes. As características desta amostra podem ser consultadas no Quadro I.

Após a aleatorização destes utentes foram obtidos dois grupos: o primeiro, que teve como intuito a elaboração do modelo preditivo, foi composto por 329 elementos; o segundo, destinado à validação interna do modelo obtido, foi constituído por 164 utentes. As características destes dois grupos estão descritas no Quadro II.

\begin{tabular}{|c|c|}
\hline \multicolumn{2}{|c|}{$\begin{array}{l}\text { QUADRO I. Características da amostra - modelação } \\
\text { + validade interna }(n=493)\end{array}$} \\
\hline \multicolumn{2}{|l|}{ USF } \\
\hline - Eça & $55,6 \%$ \\
\hline$-\mathrm{CSI}$ & $44,4 \%$ \\
\hline \multicolumn{2}{|l|}{ Género } \\
\hline - Feminino & $64,3 \%$ \\
\hline - Masculino & $35,7 \%$ \\
\hline Idade (anos) & $57,7 \pm 17,2^{\mathrm{a}}$ \\
\hline Altura $(\mathrm{cm})$ & $162,0 \pm 9,5^{a}$ \\
\hline Peso (kg) & $71,8 \pm 14,2^{a}$ \\
\hline $\mathrm{IMC}\left(\mathrm{kg} / \mathrm{m}^{2}\right)$ & $27,4 \pm 4,6^{a}$ \\
\hline Perímetro abdominal $(\mathrm{cm})$ & $93,8 \pm 11,9^{a}$ \\
\hline
\end{tabular}

a média \pm desvio-padrão; IMC: índice de massa corporal; USF: Unidade de Saúde Familiar

\begin{tabular}{|c|c|c|c|}
\hline \multicolumn{4}{|c|}{$\begin{array}{l}\text { QUADRO II. Comparação entre as amostras de moc } \\
\text { interna }\end{array}$} \\
\hline & \multicolumn{2}{|c|}{ Amostras } & \multirow[t]{2}{*}{ Valor $p$} \\
\hline & $\begin{array}{l}\text { Modelação } \\
(n=329)\end{array}$ & $\begin{array}{l}\text { Validação Interna } \\
\qquad(n=164)\end{array}$ & \\
\hline \multicolumn{4}{|l|}{ Género } \\
\hline - Feminino & $66,3 \%$ & $60,4 \%$ & $0,23 *$ \\
\hline - Masculino & $33,7 \%$ & $39,6 \%$ & \\
\hline Idade (anos) & $58,4 \pm 17,1^{a}$ & $56,2 \pm 17,2^{a}$ & $0,17^{* *}$ \\
\hline Altura $(\mathrm{cm})$ & $161,7 \pm 9,4^{a}$ & $162,5 \pm 9,6^{a}$ & $0,37^{* *}$ \\
\hline Peso (kg) & $72,0 \pm 14,0$ & $71,4 \pm 14,4$ & $0,65^{* *}$ \\
\hline $\mathrm{IMC}\left(\mathrm{kg} / \mathrm{m}^{2}\right)$ & $27,6 \pm 4,5^{\mathrm{a}}$ & $27,0 \pm 4,6^{a}$ & $0,19 * *$ \\
\hline Perímetro abdominal $(\mathrm{cm})$ & $94,1 \pm 11,9^{a}$ & $93,1 \pm 12,1^{\mathrm{a}}$ & $0,36^{* *}$ \\
\hline
\end{tabular}

a média \pm desvio-padrão; IMC: índice de massa corporal; *teste $\chi_{\text {Pearson; }}^{2}{ }^{* *}$ teste $t$

A amostra de modelação apresentou uma idade média de 58,4 $\pm 17,1$ anos, com idades compreendidas entre os 20 e os 95 anos. Foi observada uma altura média de $161,7 \pm 9,4$ cm com um mínimo de $135 \mathrm{~cm}$ e um máximo de $185 \mathrm{~cm}$. Os elementos desta amostra tinham um peso médio de $72,0 \pm 14,0 \mathrm{~kg}$ variando entre os $40 \mathrm{~kg}$ e os $126 \mathrm{~kg}$. O IMC médio foi de $27,6 \pm 4,5 \mathrm{~kg} / \mathrm{m}^{2}$, com um mínimo de $17,8 \mathrm{~kg} / \mathrm{m}^{2}$ e um máximo de $42,2 \mathrm{~kg} / \mathrm{m}^{2}$. A maioria dos participantes era do género feminino $(66,3 \%)$.

A amostra de validação interna apresentou uma idade média de 56,2 $\pm 17,2$ anos, com idades compreendidas entre os 20 e os 91 anos. Foi observada uma altura média de 162,5 \pm 9,6cm com um mínimo de $144 \mathrm{~cm}$ e um máximo de $187 \mathrm{~cm}$. Os elementos desta amostra tinham um peso médio de $71,4 \pm 14,4 \mathrm{~kg}$ variando entre os $40 \mathrm{~kg}$ e os $119 \mathrm{~kg}$. O IMC médio foi de $27,0 \pm 4,6 \mathrm{~kg} / \mathrm{m}^{2}$, com um mínimo de $16,2 \mathrm{~kg} / \mathrm{m}^{2}$ e um máximo de $44,0 \mathrm{~kg} / \mathrm{m}^{2}$. A maioria dos participantes era do género feminino $(60,4 \%)$.

Não foram encontradas diferenças significativas entre as amostras de modelação e de validação interna pelo que estas foram consideradas homogéneas (Quadro II).

A segunda amostra, destinada à validação externa do modelo, foi constituída por 240 utentes. Esta apresentou uma idade média de $60,8 \pm 14,7$ anos, com ida- 


\begin{tabular}{|c|c|c|c|}
\hline & \multicolumn{2}{|c|}{ Amostras } & \multirow[t]{2}{*}{ Valor $\mathbf{p}$} \\
\hline & $\begin{array}{l}\text { Modelação } \\
(n=329)\end{array}$ & $\begin{array}{l}\text { Validação Externa } \\
\qquad(n=240)\end{array}$ & \\
\hline \multicolumn{4}{|l|}{ Género } \\
\hline - Feminino & $66,3 \%$ & $61,2 \%$ & $0,22^{*}$ \\
\hline - Masculino & $33,7 \%$ & $38,8 \%$ & \\
\hline Idade (anos) & $58,4 \pm 17,1^{\mathrm{a}}$ & $60,8 \pm 14,7^{a}$ & $0,08^{* *}$ \\
\hline Altura $(\mathrm{cm})$ & $161,7 \pm 9,4^{a}$ & $161,5 \pm 8,5^{a}$ & $0,37^{* *}$ \\
\hline Peso (kg) & $72,0 \pm 14,0$ & $71,0 \pm 13,8$ & $0,80 * *$ \\
\hline $\mathrm{IMC}\left(\mathrm{kg} / \mathrm{m}^{2}\right)$ & $27,6 \pm 4,5^{\mathrm{a}}$ & $27,2 \pm 4,7^{\mathrm{a}}$ & $0,36 * *$ \\
\hline Perímetro abdominal $(\mathrm{cm})$ & $94,1 \pm 11,9^{a}$ & $94,8 \pm 11,6^{a}$ & $0,50 * *$ \\
\hline
\end{tabular}

${ }^{a}$ média \pm desvio-padrão; IMC: índice de massa corporal; ${ }^{*}$ teste $\chi^{2}{ }_{\text {Pearson }}{ }^{* *}$ teste $t$
Foram verificados os pressupostos teóricos de aplicabilidade do modelo (normalidade, homocedasticidade, inexistência de correlação serial, inexistência de multicolinearidade e ausência de observações influentes). O modelo mostrou ter um $\mathrm{R}^{2}$ de 0,772 e um erro-padrão de $5,71 \mathrm{~cm}$, como se descreve em maior detalhe no Quadro IV. A variável mais importante para a determinação do PA foi o IMC com um $\mathrm{R}^{2}$ de 0,641 indicando que, por cada unidade de aumento do IMC, o PA aumenta em média $2,019 \mathrm{~cm}$. O modelo mostrou também que a variável altura é um preditor independente do PA, aumentando este $0,327 \mathrm{~cm}$ por cada centímetro de altura. O modelo mostrou ainda que o PA aumenta com a idade, estimando um des compreendidas entre os 21 e os 93 anos. Foi observada uma altura média de $161,5 \pm 8,5 \mathrm{~cm}$ com um mínimo de $142 \mathrm{~cm}$ e um máximo de $188 \mathrm{~cm}$. Os elementos desta amostra tinham um peso médio de $71,0 \pm 13,8 \mathrm{~kg}$ variando entre os $43 \mathrm{~kg}$ e os $118 \mathrm{~kg}$. O IMC médio foi de $27,2 \pm 4,7 \mathrm{~kg} / \mathrm{m}^{2}$, com um mínimo de $17,2 \mathrm{~kg} / \mathrm{m}^{2}$ e um máximo de $42,7 \mathrm{~kg} / \mathrm{m}^{2}$. A maioria dos participantes era do género feminino (61,2\%). A comparação com a amostra de modelação não revelou diferenças significativas (Quadro III).

\section{Modelo de Regressão Linear}

Foram introduzidas no modelo as variáveis idade, género, altura, massa corporal, PA e IMC. Exceptuando a variável massa corporal, todas as variáveis introduzidas no modelo foram significativas, tendo-se obtido o seguinte modelo preditivo do PA:

$$
\begin{gathered}
\mathrm{PA}=2,019^{*} \mathrm{IMC}+0,211^{*}(\text { Idade }-20) \\
+0,327^{*} \text { Altura }-1,951^{*} \text { Género }-21,031
\end{gathered}
$$

em que Género é uma variável dicotómica que toma o valor 0 se masculino e 1 se feminino.

A variável massa corporal foi excluída do modelo por apresentar elevada colinearidade com as variáveis altura e IMC (Tolerância $=0,011$; Factor de Inflação da Variância = 92,96). incremento de $0,211 \mathrm{~cm}$ por cada ano. Por fim verificou-se que o género feminino apresenta em média um $1,951 \mathrm{~cm}$.

\section{Validades Interna e Externa}

A aplicação do modelo à amostra de validade interna mostrou que 152 casos $\left(92,7 \%\right.$; $\mathrm{IC}_{95 \%}: 88,7 \%$ - 96,7\%) foram correctamente preditos pelo modelo. $\mathrm{O}$ mesmo modelo aplicado à amostra de validação externa previu correctamente o PA de 225 casos $\left(93,8 \%\right.$; $\mathrm{IC}_{95 \%}: 90,7 \%$ - 96,8\%), não diferindo estas percentagens de forma significativa $\left(\chi_{\text {Pearson(1) }}^{2}=0,178 ; \mathrm{p}=0,67\right)$.

\begin{tabular}{|c|c|c|c|c|}
\hline \multicolumn{5}{|c|}{ QUADRO IV. Modelo preditivo do perímetro abdominal } \\
\hline Modelo & Coeficiente $\beta$ & Erro-padrão & valor $p$ & $\mathbf{R}^{2}$ parcial \\
\hline Constante & $-21,031$ & 8,949 & 0,019 & - \\
\hline IMC & 2,019 & 0,072 & $<0,001$ & 0,641 \\
\hline Género & $-1,951$ & 0,904 & 0,032 & 0,055 \\
\hline Idade - 20 & 0,211 & 0,021 & $<0,001$ & 0,044 \\
\hline Altura & 0,327 & 0,049 & $<0,001$ & 0,032 \\
\hline
\end{tabular}
PA inferior ao do género masculino em cerca de

IMC: índice de massa corporal 


\section{DISCUSSÃO}

Os resultados deste estudo mostraram que as variáveis IMC, género, idade e altura de um utente podem ser incluídas num modelo preditivo que permite estimar com precisão o seu PA.

Neste estudo o IMC foi a variável com maior influência na determinação do PA, ou seja, quanto maior o IMC do utente maior deverá ser o seu PA. Este resultado está de acordo com outros estudos que encontraram uma forte correlação entre o IMC e o PA., ${ }^{4,12,16}$ As correlações encontradas, tanto no nosso estudo como noutros anteriormente publicados, não são perfeitas, o que pode ser explicado, por um lado, por o IMC não representar apenas a massa gorda, ${ }^{3}$ por outro, por ser necessário ter em conta outras variáveis que influenciam o valor do PA.

Um aspecto interessante deste estudo foi o ter mostrado que a altura do utente tem influência no PA. De facto, é concebível que uma pessoa mais alta apresente um maior PA sem que este esteja, necessariamente, associado a maior obesidade visceral. Esta relação foi demonstrada por Heymsfield e colaboradores, ou seja, indivíduos mais altos apresentam perímetros abdominais superiores quando comparados com indivíduos com estaturas inferiores, e que esta relação se torna ainda mais evidente após ajustamento para outras variáveis. ${ }^{17}$ Schneider e colaboradores consideram que o estabelecimento de um ponto de corte para o PA sem ter em conta a altura do indivíduo pode subestimar a quantidade relativa de adiposidade abdominal em indivíduos de baixa estatura e sobrestimar nos indivíduos mais altos. ${ }^{7}$ Como solução para este problema, vários estudos têm apontado a determinação da razão entre o PA e a altura (waist-to-height ratio, WHtR) como um melhor indicador de risco cardiovascular que o IMC ou que o PA isoladamente. ${ }^{4,6,7,18}$ Este problema foi tido em conta nas normas de orientação clínica da ESC, que recomenda que a avaliação do PA tenha em atenção a altura do utente na determinação do risco cardiovascular. ${ }^{8}$

Neste estudo verificou-se que o género influencia o PA, isto é, para o mesmo IMC, altura e idade, um homem apresenta em média mais $1,951 \mathrm{~cm}$ de PA. A existência desta diferença está de acordo com as recomendações da ESC ${ }^{8}$ que recomenda valores diferentes de PA consoante o género, devendo os utentes ser aconselhados a reduzir o seu peso caso o PA seja $\geq 102 \mathrm{~cm}$ no género masculino ou $\geq 88 \mathrm{~cm}$ no género feminino. No entanto, verifica-se que a diferença entre estes dois valores é de $14 \mathrm{~cm}$ ao passo que no nosso modelo a diferença é de apenas cerca de $2 \mathrm{~cm}$. Uma possível explicação para esta discrepância relaciona-se com a inclusão da altura no modelo. De facto, o género masculino apresenta, em média, na idade adulta, uma altura superior ao género feminino, um efeito já incluído no modelo proposto. Se tivermos como referência as curvas de crescimento da Direcção-Geral da Saúde, ${ }^{19}$ verificamos que o percentil 50 aos 20 anos no género masculino é $177 \mathrm{~cm}$ para a estatura e $23,0 \mathrm{~kg} / \mathrm{m}^{2}$ para o IMC, ao passo que no género feminino é $163 \mathrm{~cm}$ e $21,8 \mathrm{~kg} / \mathrm{m}^{2}$ respectivamente. Se estas diferenças de $14 \mathrm{~cm}$ na estatura e de $1,2 \mathrm{~kg} / \mathrm{m}^{2}$ no IMC forem aplicadas no modelo verifica-se uma diferença média de PA de 2,019 * 1,2 + 0,327 ${ }^{*} 14=7,0 \mathrm{~cm}$ entre os dois géneros. Ou seja, em conjunto, as diferenças de altura, IMC e género representariam uma diferença média de 7,0 $+1,95=8,95 \mathrm{~cm}$. Estes resultados mostram que é mais difícil alcançar o objectivo preconizado pelas normas das ESC no género feminino no que diz respeito ao PA.

Os resultados deste estudo mostraram ainda que o PA tem tendência a aumentar com a idade, ou seja, utentes mais idosos apresentam tendencialmente maior PA. De facto, de acordo com este modelo, o PA aumenta cerca de $1 \mathrm{~cm}$ a cada 5 anos, relação esta que é independente do género, altura ou IMC do indivíduo. Assim, é de esperar que seja mais difícil atingir os objectivos para o PA no utente mais idoso. Esta relação ganha ainda maior importância se tivermos em conta que a própria idade é considerada um factor de risco independente para o risco cardiovascular. De acordo com as normas de ESC, o risco está aumentado no homem a partir dos 55 anos, e na mulher a partir dos 65 anos. ${ }^{8}$ Estes resultados parecem indicar a existência de um mecanismo subjacente que justifique o aumento concomitante do perímetro abdominal com a idade. Uma possível explicação para este fenómeno poderá estar relacionada com a sarcopenia associada à idade, ou seja, a redução da massa muscular esquelética que ocorre mesmo no idoso saudável. ${ }^{20,21}$ Este fenómeno parece ter natureza multifactorial, estando relacionado com alterações na enervação central e periférica, modificações hormonais e inflamatórias com efeitos catabólicos a nível muscular, e reduções na ingesta calórica e protei- 
ca, que se revelam mais pronunciados no género masculino. ${ }^{21} \mathrm{~A}$ diminuição da massa muscular esquelética induz uma diminuição da taxa metabólica basal, que no adulto jovem pode ser responsável por 60 a $80 \%$ do gasto energético diário. ${ }^{22}$ Apesar de ocorrer redução da ingesta calórica com a idade, esta é desproporcionada relativamente à perda muscular, resultando em aumento da massa gorda corporal ${ }^{22}$ onde se inclui o tecido adiposo visceral associado ao aumento do PA. Este mecanismo reforça a importância da modificação dos estilos de vida, nomeadamente uma alimentação correcta e prática regular de exercício físico, na redução do risco cardiovascular associado à idade.

De todas as variáveis com potencial influência no PA avaliadas neste estudo, a única passível de ser intervencionada é o IMC, através da modificação da massa corporal. Os resultados deste estudo mostraram que o conhecimento das características do utente pode ser utilizado para estimar o PA. O modelo mostrou ter excelente validade interna e externa, deixando antever a sua potencial aplicabilidade em contexto clínico através da individualização do plano terapêutico, mais concretamente no estabelecimento de metas de redução ponderal com vista a reduzir o risco cardiovascular.

Devem ser reconhecidas algumas limitações deste estudo. Trata-se de uma amostra de conveniência pelo que a generalização dos resultados deve ser feita com cautela. Optámos por uma amostra de conveniência por se tratar de uma primeira abordagem a esta linha de investigação em que tivemos a intenção de verificar se seria possível prever o PA com base no conhecimento de algumas características do utente. Reconhecemos também que o estudo não considerou outras variáveis com potencial explicativo do PA, como por exemplo a raça, ${ }^{8,17}$ estado de saúde ${ }^{17}$ ou o consumo de álcool. A variabilidade do modelo poderá ter sido influenciada pelos diferentes instrumentos de medida disponíveis em cada consultório médico bem como pela medição única das variáveis em estudo.

Em conclusão, este estudo mostrou que é possível prever o PA com base no conhecimento das características individuais do utente. A utilização deste tipo de modelos na prática clínica poderá facilitar o aconselhamento objectivo e individualizado e em última instância resultar numa melhor abordagem da redução ponderal e consequente redução do risco cardiovascular.

\section{REFERÊNCIAS BIBLIOGRÁFICAS}

1. Instituto Nacional de Estatística Óbitos por causa de morte. Disponível em: http://www.ine.pt/xportal/xmain?xpid=INE\&xpgid=ine_indicadores\&indOcorrCod $=0001675 \&$ contexto $=b d \&$ selTab $=$ tab2\&xlang =pt [acedido em 15/01/2012].

2. Phillips LK, Prins JB. The link between abdominal obesity and the metabolic syndrome. Curr Hypertens Rep 2008 Apr; 10 (2): 156-64.

3. Taylor AE, Ebrahim S, Ben-Shlomo Y, Martin RM, Whincup PH, Yarnell $J W$, et al. Comparison of the associations of body mass index and measures of central adiposity and fat mass with coronary heart disease, diabetes, and all-cause mortality: a study using data from 4 UK cohorts. Am J Clin Nutr 2010 Mar; 91 (3): 547-56.

4. Gruson E, Montaye M, Kee F, Wagner A, Bingham A, Ruidavets JB, et al. Anthropometric assessment of abdominal obesity and coronary heart disease risk in men: the PRIME study. Heart 2010 Jan; 96 (2): 136-40.

5. Kershaw EE, Flier JS. Adipose tissue as an endocrine organ. J Clin Endocrinol Metab 2004 Jun; 89 (6): 2548-56.

6. Haun DR, Pitanga FJ, Lessa I. Razão cintura-estatura comparado a outros indicadores antropométricos de obesidade como preditor de risco coronariano elevado. Rev Assoc Med Bras 2009 Nov-Dec; 55 (6): $705-$ 11.

7. Schneider HJ, Friedrich N, Klotsche J, Pieper L, Nauck M, John U, et al. The predictive value of different measures of obesity for incident cardiovascular events and mortality. J Clin Endocrinol Metab 2010 Apr; 95 (4): 1777-85.

8. Graham I, Atar D, Borch-Johnsen K, Boysen G, Burell G, Cifkova R, et al. European guidelines on cardiovascular disease prevention in clinical practice: executive summary: Fourth Joint Task Force of the European Society of Cardiology and Other Societies on Cardiovascular Disease Prevention in Clinical Practice (Constituted by representatives of nine societies and by invited experts). Eur Heart J 2007 Oct; 28 (19): 2375414.

9. Lee CM, Huxley RR, Wildman RP, Woodward M. Indices of abdominal obesity are better discriminators of cardiovascular risk factors than BMI: a meta-analysis. J Clin Epidemiol 2008 Jul; 61 (7): 646-53.

10. Wu HY, Xu SY, Chen LL, Zhang HF. Waist to height ratio as a predictor of abdominal fat distribution in men. Chin J Physiol 2009 Dec 31; 52 (6): 441-5.

11. Goodpaster BH, Delany JP, Otto AD, Kuller L, Vockley J, South-Paul JE, et al. Effects of diet and physical activity interventions on weight loss and cardiometabolic risk factors in severely obese adults: a randomized trial. JAMA 2010 Oct 27; 304 (16): 1795-802.

12. Stevens J, Katz EG, Huxley RR. Associations between gender, age and waist circumference. Eur J Clin Nutr 2010 Jan; 64 (1): 6-15.

13. Microsoft. Microsoft Excel [computer software]. RedmondWashington: Microsoft; 2003.

14. R Development Core Team. R: a language and environment for statistical computing. Vienna: R Foundation for Statistical Computing; 2009.

15. SPSS 15.0 for Windows Release 15.0.0 (6 Sep 2006).

16. Camhi SM, Bray GA, Bouchard C, Greenway FL, Johnson WD, Newton $\mathrm{RL}$, et al. The relationship of waist circumference and BMI to visceral, subcutaneous, and total body fat: sex and race differences. Obesity (Silver Spring) 2011 Feb; 19 (2): 402-8.

17. Heymsfield SB, Heo M, Pietrobelli A. Are adult body circumferences as- 
sociated with height? Relevance to normative ranges and circumferential indexes. Am J Clin Nutr 2011 Feb; 93 (2): 302-7.

18. Schneider HJ, Klotsche J, Silber S, Stalla GK, Wittchen HU. Measuring abdominal obesity: effects of height on distribution of cardiometabolic risk factors risk using waist circumference and waist-to-height ratio. Diabetes Care 2011 Jan; 34 (1): e7.

19. Direção-Geral da Saúde. Circular Normativa Nº: 05/DSMIA. Consultas de Vigilância de Saúde Infantil e Juvenil, Actualização das curvas de crescimento, 2006. Disponível em: http://www.onocop.pt/conteudos/documentos/Percentis_SIJ.pdf [acedido em 15/01/2012].

20. Boneva-Asiova Z, Boyanov M. Age-related changes of body composition and abdominal adipose tissue assessed by bio-electrical impedance analysis and computed tomography. Endocrinol Nutr 2011 Nov; 58 (9): 472-7.

21. Doherty TJ. Invited review: Aging and sarcopenia. J Appl Physiol 2003 Oct; 95 (4): 1717-27.
22. van Pelt RE, Dinneno FA, Seals DR, Jones PP. Age-related decline in RMR in physically active men: relation to exercise volume and energy intake. Am J Physiol Endocrinol Metab 2001 Sep; 281 (3): E633-9.

\section{CONFLITOS DE INTERESSES}

O autor Frederico Rosário é revisor convidado da Revista Portuguesa de Clínica Geral e declara não ter estado envolvido no processo de decisão editorial para este artigo.

\section{ENDEREÇO PARA CORRESPONDÊNCIA}

Frederico Rosário

Centro de Saúde da Quinta da Lomba, Rua Jornal Heraldo

2830-195 Barreiro

Recebido em 27/09/2011

Aceite para publicação em 21/11/2011

\section{ABSTRACT}

\section{DERIVATION AND VALIDATION OF A MODEL FOR PREDICTING WAIST CIRCUMFERENCE}

Objective: To analyze the relationship between waist circumference and the age, gender, height, and weight of patients, and to design a model to predict waist circumference using these variables.

Type of study: Observational

Setting: Eça Family Health Unit (FHU), Cuidados de Saúde Integrados Seixal and Quinta da Lomba. Health Care Center (HCC) Population: Patients aged 20 years or older attending medical consultations in these units.

Methods: A convenience sample of patients was drawn from patients who attended medical consultations at the FHU in May and July 2009, and the HCC between February and April 2010. Data collected from patients included age, height, weight, gender, and waist circumference. The population at the FHU was randomly divided into two groups. Data obtained from two thirds of the patients were used for developing the model and data from the remaining one third were used to determine the internal validity of the model. The model design was based on a multiple linear regression model, using the stepwise method, with a $5 \%$ entry probability and a $10 \%$ removal probability. The sample of patients from the HCC was used for external validation of the model.

Results and Conclusions: The FHU sample included 493 patients, of these $55,6 \%$ were from USF Eça, and $64,3 \%$ were female. The average age of patients in the sample was $57,7 \pm 0,8$ years, the mean height was $162 \pm 0,4 \mathrm{~cm}$, the mean BMI was $27,3 \pm 0,2 \mathrm{~kg} / \mathrm{m}^{2}$, and the mean waist circumference was $93,8 \pm 0,5 \mathrm{~cm}$. Comparison of the modeling and internal validation groups revealed no significant differences between them. A model for the prediction of waist circumference was designed. All predictor variables were significantly associated with the outcome variable with an $R^{2}=0,772$. The model showed an internal validity of $92,7 \%$. Applying the model to the external validation group, consisting of 240 patients, showed that the model predicted the waist circumference of $93,8 \%$ of these patients within the $95 \%$ confidence interval predicted by the model. In conclusion height, weight, age and gender can be used to accurately estimate waist circumference.

Keywords: Waist Circumference; Risk; Cardiovascular Diseases; Linear Models. 